\title{
Philosophiques
}

\section{Andler (éd.), Introduction aux sciences cognitives, Paris, Folio, coll. « Essais », 1992, 516 pages.}

\section{Luc Faucher}

Volume 21, numéro 1, printemps 1994

URI : https://id.erudit.org/iderudit/027262ar

DOI : https://doi.org/10.7202/027262ar

Aller au sommaire du numéro

Éditeur(s)

Société de philosophie du Québec

ISSN

0316-2923 (imprimé)

1492-1391 (numérique)

Découvrir la revue

Citer ce compte rendu

Faucher, L. (1994). Compte rendu de [D. Andler (éd.), Introduction aux sciences cognitives, Paris, Folio, coll. « Essais », 1992, 516 pages.] Philosophiques, 21(1),

262-267. https://doi.org/10.7202/027262ar d'utilisation que vous pouvez consulter en ligne.

https://apropos.erudit.org/fr/usagers/politique-dutilisation/ 
D. Andler (éd.), Introduction aux sciences cognitives, Paris, Folio, coll. "Essais ", 1992, 516 pages.

\section{par Luc Faucher}

Une remarque s'impose d'entrée de jeu à propos de cet ouvrage : son titre est trompeur. On pourrait légitimement s'attendre, avec un tel titre, à une introduction didactique aux sciences cognitives; or le recueil ne contient ni description systématique des méthodes et domaines d'investigation propres à 
ce paradigme, ni contribution classique aux débats qui ont jalonné son histoire. Il est constitué principalement de communications présentées lors d'un colloque à Cerisy-la-Salle en r 987 auxquelles se sont greffés des textes inédits ou déjà parus ailleurs. Le caractère circonstanciel des contributions procure toutefois un excellent aperçu des tendances actuelles en SC (abréviation pour sciences cognitives).

Depuis quelques années, on assiste à la montée irrésistible d'un nouveau type de modélisation qui prétend permettre une simulation plus réaliste des performances intelligentes que celle offerte par le modèle computationaliste classique. En résolvant des problèmes non-résolus dans le cadre du computationalisme, le connexionnisme place ce dernier en situation de crise et s'impose comme le nouveau paradigme des SC. Tout ceci ne se fait pas sans résistance et le débat fait actuellement rage autour du caractère véritablement révolutionnaire du connexionnisme. Reflet des controverses actuelles, la question des relations entre les deux types de modèle est le sujet d'un grand nombre de communications. Parmi celles-ci, il faut d'abord consulter le texte de Smolensky, «IA connexionniste, I A symbolique et cerveau », qui est essentiel à la compréhension du connexionnisme. Selon ce dernier, les modélisations connexionnistes des processus cognitifs s'inscrivent dans ce qu'il nomme le paradigme subsymbolique qu'il compare au paradigme symbolique dominant en SC. Dans le paradigme symbolique, les structures mentales décrites par la psychologie ordinaire sont formalisées en termes de langage de la pensée qui repose lui-même sur des niveaux d'implémentations physiques inférieurs. Le connexionnisme ajoute un niveau d'interprétation intermédiaire entre le niveau neurologique et symbolique, soit le niveau subsymbolique. On peut décrire ces systèmes à deux niveaux : à un niveau inférieur oủ les variables sont dynamiques (définies en termes de niveau d'activation et de force de connexion) et un niveau supérieur où les représentations sont émergentes et statistiques (schèmes).

$\mathrm{Si}$ le texte de Smolensky permet de comprendre les différences entre les deux types de modèles, celui de Cummins et Schwartz, « Connexionnisme, computation et cognition $\gg$, situe le connexionnisme dans le projet des SC. Ce qu'entendent démontrer les auteurs, c'est que même si le connexionnisme partage généralement les hypothèses du computationalisme, il existe une forme de connexionnisme radical qui s'en démarque par le rejet de la thèse centrale du computationalisme, soit le représentationalisme. Une grande partie des recherches connexionnistes est liee à cette thèse. Cependant, cette connexion n'est pas necessaire. Une architecture connexionniste pourrait régir non-computationellement les transitions d'états représentationnels. Cette possibilité repose sur l'existence de fonctions cognitives non calculables, non régies par des règles. La conséquence de ce type de connexionnisme serait de faire disparaître la possibilité de définir la cognition autrement que par ostension, par exemple comme ce que les humains font lorsqu'ils résolvent un problème.

Dreyfus, dans « La portée philosophique du connexionnisme », prétend pour sa part démontrer la supériorité du connexionnisme sur le computationalisme quand vient le temps d'expliquer les performances dépendant de la possession d'une expertise. Il existe une longue tradition, qui va de Socrate jusqu'à l'intelligence artificielle classique, qui veut que comprendre un 
domaine particulier d'activité, c'est posséder une theorie sur celui-ci. Ainsi l'expert dans un domaine est celui qui, par induction, en est venu à formuler un ensemble de règles subsumant ce domaine (p.e. les règles de production de Simon). Dreyfus s'oppose à ce modèle inductif de l'acquisition des connaissances et propose un processus inverse pour expliquer l'acquisition de l'expertise. Selon lui, l'apprentissage dans certains domaines débute par l'acquisition de règles a-contextuelles et se termine au dernier niveau, par la possession de l'expertise, caractérisée par le fait que le sujet n'applique plus de règles. Le connexionnisme proposant justement des systèmes n'utilisant pas de règles, celui-ci pourrait donc permettre d'expliquer l'acquisition de l'expertise en postulant l'utilisation des détecteurs de traits.

Le dernier texte portant sur le connexionnisme, celui de Anspach et Varela ( Le système immunitaire : un soi cognitif autonome $»$ ), me semble doublement intéressant. D'abord, il fournit un exemple de systèmes cognitifs noncomputationels dont parlent Cummins et Schwartz; mais surtout, il propose une approche révolutionnaire de l'immunologie qui ne pourra manquer d'avoir un effet de retour sur la question du caractère inné des concepts (puisque l'exemple de l'immunologie est souvent invoqué par les innéistes). Il ne fait pas de doute pour les auteurs que le système immunitaire est un système cognitif dont les propriétés émergent de réseaux analogues à ceux des systèmes connexionnistes. Cette idée s'accorde avec les nouvelles données de l'immunologie qui montrent que le caractère prométhéen du système immunitaire ne peut s'expliquer en termes de programmation génétique. Dans la théorie qu'ils développent, l'antigène cesse d'être déterminant et devient une légère perturbation dans le réseau. Le rôle du système immunitaire n'est plus que de maintenir l'équilibre entre les constituants du soi et les antigènes. Les auteurs sont donc conduits à soutenir cette surprenante conclusion : le rôle défensif du système immunitaire n'est qu'un cas limite de l'affirmation de l'identité moléculaire.

Une autre question qui occupe l'avant-scène des SC, et qui est largement représentée dans le recueil, est celle de la modularité. Cette thèse affirme que les processus périphériques sont inférentiels comme la cognition, mais sont encapsulés, rapides et imperméables à l'influence des processus de haut niveau comme les réflexes. Plusieurs textes posent la question de la signification de cette thèse et tentent d'établir le degré d'encapsulation du traitement de l'information de bas niveau (par exemple, les textes de Colthheart et Davies, Segui). Du lot, c'est celui de Treisman, « L'attention, les traits et la perception des objets », qui mérite le plus d'attention, surtout parce qu'il suggère une explication très convaincante du rôle de l'attention dans la cognition. Selon Treisman, les processus à l'œuvre dans les premières étapes du traitement de l'information visuelle sont caractérisées par leur facilité, leur autonomie et leur modularité. Cette caractérisation permet à Treisman de faire l'hypothèse que les traits qui permettent le pop-out (le fait qu'un trait saute aux yeux) sont perçus par des modules autonomes. Selon Treisman, les traits sont enregistrés séparément par les modules et l'attention focalisée serait nécessaire pour conjoindre plusieurs types de traits (dans ce cas, l'effet de pop-out disparait). Des expériences montrent également que la connaissance de certaines conjonctions de traits peut réduire les erreurs d'identification. Ces effets 
semblent s'expliquer, non pas par l'imposition de « frame » sur les perceptions (effets top-doun), mais plutôt par le fait que les conjonctions temporaires sont comparées aux objets en mémoire. Cette hypothèse force Treisman à supposer l'existence de représentations temporaires indépendantes de leurs étiquettes et à poser une distinction entre voir et identifier.

La distinction introduite par Treisman nous permet d'introduire cet autre thème important des SC, celui de l'acquisition des concepts. Dans « Un modèle à deux étapes de la formation des concepts », Woodfield nous propose une expérience de pensée visant à répondre à trois problèmes : I) le problème du petit savant, qui se pose lorsque l'on se représente l'acquisition de concepts comme un processus de construction et de mise à l'épreuve d'hypothèses. Le problème est que pour former ainsi des hypothèses sur certains concepts, ceux-ci doivent déjà être présents chez l'enfant, laissant leur formation inexpliquée. 2) Le problème du holisme : l'identité de chaque concept étant fixée par ses relations avec les autres, comment un réseau holiste peut-il se développer? 3) Le problème de l'attribution de contenu aux concepts à létat embryonnaire: selon les conditions proposées par Stich (la ressemblance fonctionnelle et la ressemblance idéologique), les enfants et les adultes ne partagent pas les mêmes concepts. Quel contenu peut-on alors attribuer aux états mentaux des enfants? La solution à ces problèmes passe par l'étude du processus mis en jeu par le développement conceptuel, plus particulièrement celui de la caractérisation perceptuelle. Selon Woodfield, la caractérisation exige le travail d'un catégorisateur dont la fonction est de subsumer les objets sous un schème. Ce sont ces schèmes perceptuels qu'utilise l'intellect pour se développer. La thèse de Woodfield repose sur l'hypothèse fodorienne qui fait de l'intellect (de l'enfant) un manipulateur de symboles internes (symboles qui ne possèdent aucune valeur sémantique au départ). Le but de Woodfield est d'expliquer comment ces symboles peuvent en venir à jouer le rôle de nos concepts. Pour ce faire, il a recours à la notion de protoconcept, structure qui peut se remplir peu à peu de phrases, jusqu'à ce que son contenu en vienne à ressembler au nôtre, et puisse être identifié à un concept.

Les tentatives des chercheurs en SC pour produire des programmes permettant à des ordinateurs d'exhiber un comportement verbal et une compréhension similaire aux nôtres ont jusqu'ici échoué. Pour plusieurs, c'est faute d'avoir négligé la dimension pragmatique de la communication. Quelques textes du présent volume tentent d'élaborer différents aspects de cette théorie pragmatique de la communication (Kayser, Récanati et Livet). Wilson et Sperber, dans « Ressemblance et communication », suggèrent une alternative a la tradition qui oppose les représentations en fonction de la ressemblance et les représentations conventionnelles (comme les représentations linguistiques). Selon la tradition, une règle de littéralité est exigée pour comprendre ce que quelqu'un veut dire en employant une phrase déclarative. Une proposition est vue comme étant identique à la pensée que l'on veut exprimer par elle. Dans ce type d'analyse, la métaphore et l'ironie sont considérées comme des écarts vis-à-vis d'une règle de littéralité. L'alternative de Sperber et Wilson consiste à exploiter plutôt la notion de ressemblance entre la pensée et l'énoncé. Comme les énoncés et les pensées ont tous deux des propriétés logico-sémantiques, il est possible d'utiłiser un énoncé pour représenter une 
pensée. L'important est qu'un énoncé partage certaines propriétés d'une pensée. Il reste alors à expliquer comment l'auditeur peut comprendre un locuteur sans utiliser la règle de littéralité. Les auteurs supposent que nous utilisons le principe de pertinence (PP), sorte de maxime pragmatique selon laquelle « tout énoncé communique au destinataire la présomption de sa pertinence optimale. » (p. 23o) Le PP permet donc à l'auditeur d'identifier la source de la pensée représentée et de mesurer le degré de ressemblance entre l'énoncé et la pensée.

Le problème du rapport entre le corps et l'esprit fait partie de l'héritage philosophique que nous a légué Descartes. Les tentatives d'établissement de ces rapports ont donné lieu à moult théories toutes aussi inadéquates les unes que les autres. Jacob, dans « Le problème du rapport du corps et de l'esprit aujourd'hui », entend montrer que les SC nous offrent une solution acceptable à ce problème. Il croit en effet que le fonctionnalisme concilie le matérialisme qui prédomine actuellement en science avec l'idée assez naturelle selon laquelle nos états mentaux ont un pouvoir causal. Le fonctionnalisme entraîne toutefois une complication puisqu'il faut expliquer le pouvoir des propriétés fonctionnelles (qua fonctionnelles) sur le monde afin d'éviter l'épiphènoménalisme du mental. Pour ce faire. Jacob introduit une distinction (qui ne fait pas le travail, mais qui clarifie le problème) entre les propriétés de premier ordre (qui ont un pouvoir causal) et de second ordre (qui sont fonctionnelles).

L'exploration du rapport entre les SC et les sciences sociales vient compléter l'éventail des thèmes abordés dans ce volume. Dans son texte, « Les sciences cognitives, les sciences sociales et le matérialisme », Sperber suggère l'exemple des SC et de leur matérialisme minimal afin de mettre de l'ordre dans le fouillis ontologique des sciences sociales. Il entend dépasser les positions classiques des sciences sociales, en définissant les phénomènes de ces sciences de façon à rendre manifeste la possibilité de leur existence physique. Selon lui, l'obstacle au matérialisme dans les sciences humaines vient du rôle des représentations publiques abstraites (les mythes, par exemple) qu'il faudrait pouvoir redéfinir en termes de représentations cognitives ou mentales. La solution passe, selon lui, par une épidémiologie des représentations : tout comme les virus, certaines représentations mentales se répandent dans une population par le biais de la communication, les representations les plus contagieuses deviendraient des représentations culturelles.

Dumouchel, dans « Systèmes sociaux et cognition », tente de modifier la distinction proposée par Hayek entre deux types d'ordre social lles ordres spontanés et planifiés). Pour ce faire, Dumouchel introduit une référence aux représentations des individus. A l'aide d'une fable amusante sur la création d'un ordre spontané, l'auteur en vient à proposer une typologie plus fine lui permettant d'éviter la normativité de la théorie de Hayek, qui semble confondre désirabilité sociale et ordre logique. Dumouchel substitue ainsi trois nouveaux ordres à ceux d'Hayek : l'ordre irrationnel loú les agents ne peuvent consentir aux conditions qui déterminent l'ordre sans le faire disparaître); l'ordre rationnel (où les agents peuvent consentir aux conditions sans faire disparaître l'ordre); l'ordre raisonnable (qui ne peut exister que si l'agent consent à suivre ses règles). Il faut noter que la référence à la rationalité ici ne concerne pas les agents eux-mêmes, mais plutôt les ordres sociaux. Un ordre est 
rationnel si les agents peuvent consentir aux règles qui le définissent. Elle fait également référence à la théorie des jeux et à sa notion d'équilibre ou de stabilité, permettant ainsi d'expliquer l'instabilité et la stabilité des divers ordres.

En conclusion, quoique ce livre nous présente dans l'ensemble des contributions originales et intéressantes, on peut cependant déplorer un certain nombre de lacunes qui sont dues en grande partie au fait que nous avons affaire aux actes d'un colloque. D'une part, il faut remarquer un certain manque d'unité thématique à l'intérieur des parties du livre; d'autre part, certains thèmes centraux en SC y sont ignorés, par exemple, l'imagerie mentale, l'acquisition du langage, ou les émotions. Malgré ces réserves, il faut avouer que l'ouvrage contribue à la compréhension de quelques-uns des débats récents des SC et mérite à ce titre d'être recommandé à l'attention de ceux qui s'intéressent de près ou de loin à la philosophie de la psychologie.

Département de philosophie

Université du Québec à Montréal 